FIAN/TD-06/05

ITEP/TH-22/05

\title{
Matrix Model and Stationary Problem in Toda Chain ${ }^{1}$
}

\author{
A. Marshakov \\ Lebedev Physics Institute and ITEP \\ Moscow, Russia \\ e-mail: mars@lpi.ru, mars@itep.ru
}

\begin{abstract}
We analyze the stationary problem for the Toda chain, and show that arising geometric data exactly correspond to the multi-support solutions of one-matrix model with a polynomial potential. For the first nontrivial examples the Hamiltonians and symplectic forms are calculated explicitly, and the consistency checks are performed. The corresponding quantum problem is formulated and some its properties and perspectives are discussed.
\end{abstract}

\section{Introduction}

It was found long ago (see, for example $[1,2]$ ) and emphasized recently $[3,4]$ in the context of string geometry and its certain consequences for the supersymmetric gauge theories, that the solution to one-matrix model

$$
Z=\int_{N \times N} d \Phi \exp \left(-\frac{1}{\hbar} \operatorname{Tr} W(\Phi)\right)
$$

with the polynomial potential

$$
W(\Phi)=\sum_{k=1}^{n+1} t_{k} \Phi^{k}
$$

in planar approximation within the $1 / N$-expansion is geometrically described in terms of the hyperelliptic complex curve

$$
y^{2}=W^{\prime}(\lambda)^{2}-4 f_{n-1}(\lambda)
$$

endowed with generating differential (related to the eigenvalue density for the matrix $\Phi$ )

$$
d S=y d \lambda
$$

The parameters of potential (2) in this context are certain fixed constants or "Casimirs", while the dynamical variables are rather related with nontrivial periods of generating differential (4) on hyperelliptic curve (3).

This geometric setup implies, in particular, that the most effective language for the planar solution of (1) is theory of integrable systems. Basically, already in [2] it was found that the partition function (1) $\mathcal{F}=\hbar^{2} \log Z$

\footnotetext{
${ }^{1}$ Based on talks at "Classical and quantum integrable systems", Dubna, January 2005 and "Selected topics of modern mathematical physics", St.Petersburg, June 2005, and a lecture for the minicourse: "Toda lattices: basics and perspectives", Fields Institute, Toronto, April 2005
} 
at $N \rightarrow \infty$ with fixed $\hbar N$ is geometrically formulated as a (logarithm of) quasiclassical tau-function [5]. This is a particular case of the so called algebro-geometric "holomorphic" integrable systems, quite commonly arising recently in the context of string theory or Seiberg-Witten theory (see e.g. [11, 12] and references therein).

On the other hand, it is also well-known that the matrix integral (1), as a function of parameters of the potential (2) and the size of the matrix $N$, is a tau-function of the (semi-infinite, forced) Toda chain [6]. The Toda chains are well-known families of integrable models arising in particular context of the algebro-geometric integrable systems: their affine or periodic versions are literally the Seiberg-Witten integrable systems associated to the pure ( $\mathcal{N}=2 \mathrm{SUSY}$ ) gauge theories [7]. One may be interested then, whether the integrable system, associated to (3), (4) has any relation to the Toda family.

Below we are going to demonstrate, that the complex curve (3) with the differential (4) is directly associated with a particular integrable Toda system, namely with a stationary problem in the Toda chain ${ }^{2}$. We discuss the general structure of the classical stationary problem, present explicit computations for the simplest nontrivial examples where the "order $n$ is $n=2$ and $n=3$, and discuss briefly the problems of its quantization. We hope that the observed equivalence of the matrix model and stationary Toda geometries will be first step towards understanding of proper analogs of the extra quasiclassical variables, and the considered below system can play the role of the corresponding "probe" model.

\section{Notations}

The Toda chain Lax operator is an infinite 3-diagonal matrix

$$
L_{i j}=r_{i} \delta_{j, i-1}+p_{i} \delta_{j i}+r_{i+1} \delta_{j, i+1}
$$

(with $i, j \in \mathbb{Z}$ ), where it is convenient to introduce

$$
r_{i}=e^{\frac{1}{2}\left(q_{i}-q_{i-1}\right)}
$$

or

$$
R_{i}=r_{i}^{2}=e^{q_{i}-q_{i-1}}
$$

to be useful in what follows.

The Toda chain equations of motion follow from the Lax representation

$$
\frac{\partial L}{\partial t_{1}}=\left[L, A_{1}\right]
$$

with $A_{1}=\frac{1}{2} \mathcal{R} \circ L$ or, explicitly for the $\mathcal{R}$-matrix

$$
\left(A_{1}\right)_{i j}=\frac{1}{2}\left(r_{i+1} \delta_{j, i+1}-r_{i} \delta_{j, i-1}\right)
$$

providing (8) being equivalent to the well-known equations of motion

$$
\begin{gathered}
\frac{\partial r_{i}}{\partial t_{1}}=r_{i}\left(p_{i}-p_{i-1}\right) \\
\frac{\partial p_{i}}{\partial t_{1}}=r_{i+1}^{2}-r_{i}^{2}=R_{i+1}-R_{i}
\end{gathered}
$$

for the chain of particles with the nearest neighbor exponential interaction.

In a standard way, the nonlinear equations (8) can be formulated as consistency of auxiliary linear problem

$$
(L \psi)_{i}=r_{i+1} \psi_{i+1}+p_{i} \psi_{i}+r_{i} \psi_{i-1}=\lambda \psi_{i}
$$

\footnotetext{
${ }^{2}$ Analogous stationary problem for the KdV hierarchy is well-known and widely discussed in the literature, see [8].
} 
The basis of two-dimensional space of solutions to the linear problem can be found in the form of expansion

$$
\psi_{i}^{ \pm}=\lambda^{ \pm i} e^{\mp \frac{1}{2} q_{i}}\left(1+\frac{\xi_{i}^{ \pm}}{\lambda}+\frac{\eta_{i}^{ \pm}}{\lambda^{2}}+\ldots\right)
$$

where for the coefficients of expansion one gets

$$
\begin{gathered}
\xi_{i-1}^{+}-\xi_{i}^{+}=p_{i-1} \\
\xi_{i}^{-}-\xi_{i-1}^{-}=p_{i}
\end{gathered}
$$

and

$$
\begin{gathered}
\eta_{i-1}^{+}-\eta_{i}^{+}=R_{i-1}+\xi_{i-1}^{+}\left(\xi_{i-1}^{+}-\xi_{i}^{+}\right) \\
\eta_{i}^{-}-\eta_{i-1}^{-}=R_{i+1}+\xi_{i}^{-}\left(\xi_{i}^{-}-\xi_{i-1}^{-}\right)
\end{gathered}
$$

which relates them directly with the Toda chain phase space variables.

\section{Stationary problem}

An infinite Toda chain is a completely integrable system with (infinitely many) conserving charges, which generate the "higher flows", commuting with (8). The higher flows can be written as

$$
\frac{\partial L}{\partial t_{k}}=\left[L, A_{k}\right]
$$

with the same $\mathcal{R}$-matrix $A_{k}=\frac{1}{2} \mathcal{R} \circ L^{k}$, as in (9). There are many ways to restrict the infinite Toda chain to a finite-dimensional subspace of its phase space; most well-known are $N$-periodic chain and open chain (the "Toda molecule") with $N$ particles.

Below we are going to concentrate on the stationary problem, determined by commutativity condition

$$
[L, A]=0
$$

with

$$
A=\sum_{k=1}^{n} c_{k} A_{k}
$$

for some fixed number - "order" $n$, and some coefficients $\left\{c_{k}\right\}$.

As any problem with commutativity of two operators, the stationary problem (16) acquires a complex curve

$$
\operatorname{det}(A(\lambda)-Y)=0
$$

where matrix

$$
A(\lambda)=\left(\begin{array}{ll}
a(\lambda) & b(\lambda) \\
c(\lambda) & d(\lambda)
\end{array}\right)
$$

with some polynomial matrix elements is representation of the operator (17) in the basis of two-dimensional space of the eigenfunctions (11) of the operator (5). The curve (18) is therefore hyperelliptic

$$
y^{2}=P_{2 n}(\lambda) \equiv(a-d)^{2}+4 b c
$$

with $y=2 Y-\operatorname{Tr} A(\lambda)=2 Y-a-d$.

The most natural symplectic form for the infinite Toda chain is just $\Omega_{\infty}=\sum_{i \in \mathbb{Z}} \delta q_{i} \wedge \delta p_{i}$. It is also known that it "remains intact", when reduced to the $2 N$-dimensional phase space of the $N$-periodic problem or $N$-particle open molecule, i.e. $\Omega_{N}=\sum_{i=1}^{N} \delta q_{i} \wedge \delta p_{i}$. The latter one can be also written as $\Omega_{N}=$ 
$\operatorname{res}_{w=\infty}\left(\frac{d w}{w} \operatorname{Tr}\left(\Psi^{-1} \delta L(w) \wedge \delta \Psi\right)\right)$ in terms of the spectral parameter dependent Lax operator of the $N$-periodic problem, see $[9,10,11]$ for details.

Similarly, the symplectic structure for the stationary problem can be determined by

$$
\Omega=\operatorname{res}_{\lambda=\infty}\left(d \lambda \operatorname{Tr}\left(\Psi^{-1} \delta A(\lambda) \wedge \delta \Psi\right)\right)
$$

where $\delta A(\lambda)$ is now the variation (at constant spectral parameter $\lambda$ !) of the operator (17), (19) and

$$
\Psi=\left(\begin{array}{cc}
\psi_{i}^{+} & \psi_{i}^{-} \\
\psi_{i-1}^{+} & \psi_{i-1}^{-}
\end{array}\right)
$$

is the matrix of the Baker-Akhiezer functions, with $\Psi^{-1}$ being its matrix inverse.

In what follows we will use for convenience a different from (12) normalization of the Baker-Akhiezer functions:

$$
\Psi \rightarrow \Psi\left(\begin{array}{cc}
e^{\frac{q_{i}}{2}} & 0 \\
0 & e^{-\frac{q_{i-1}}{2}}
\end{array}\right)
$$

Note, that the normalization of the Baker-Akhiezer function does not influence the symplectic form (21), since the difference, say for the change of normalization (23), is proportional to

$$
\operatorname{res}(\delta Y d \lambda) \wedge \delta\left(q_{i}-q_{i-1}\right)=0
$$

\section{Explicit computations}

Let us now illustrate the general construction by explicit computations.

\section{$4.1 \mathrm{n}=1$}

This is the trivial case of stationarity of the operator (9), which in the basis of eigenfunctions (11) reads

$$
A_{1}(\lambda)\left(\begin{array}{c}
\psi_{i} \\
\psi_{i-1}
\end{array}\right)=\left(\begin{array}{cc}
\frac{1}{2}\left(\lambda-p_{i}\right) & -r_{i} \\
r_{i} & -\frac{1}{2}\left(\lambda-p_{i-1}\right)
\end{array}\right)\left(\begin{array}{c}
\psi_{i} \\
\psi_{i-1}
\end{array}\right)
$$

giving rise to the genus zero curve (20)

$$
y^{2}=\lambda^{2}-\left(p_{i}+p_{i-1}\right) \lambda+\frac{1}{4}\left(p_{i}+p_{i-1}\right)^{2}+4 R_{i}
$$

with the expansion

$$
y d \lambda \underset{\lambda \rightarrow \infty}{\sim} d \lambda\left(\lambda-\frac{1}{2}\left(p_{i}+p_{i-1}\right)+\frac{2 R_{i}}{\lambda}+\ldots\right)
$$

The commutativity condition (16) $\left[L, A_{1}\right]=0$ leads to the further constraints

$$
\begin{gathered}
p_{i+1}=p_{i}=p_{i-1} \\
R_{i+1}=R_{i}
\end{gathered}
$$

and fixing the coefficients of (26) or (27) means that all variables turn into some constants.

In the trivial $n=1$ case, explicit calculation of (21) gives

$$
\Omega_{1}=e^{q_{i}-q_{i-1}} d q_{i} \wedge d q_{i-1}+\frac{1}{2}\left(d \xi_{i}^{+} \wedge d \xi_{i}^{-}+d \xi_{i-1}^{+} \wedge d \xi_{i-1}^{-}\right)-d \xi_{i}^{+} \wedge d \xi_{i-1}^{-}
$$

Since

$$
\frac{1}{2}\left(d \xi_{i}^{+} \wedge d \xi_{i}^{-}+d \xi_{i-1}^{+} \wedge d \xi_{i-1}^{-}\right)-d \xi_{i}^{+} \wedge d \xi_{i-1}^{-} \underset{(13)}{=} \frac{1}{2}\left(d \xi_{i}^{+} \wedge d p_{i}+d p_{i-1} \wedge d \xi_{i-1}^{-}\right)
$$

and due to (28) the symplectic form (29) vanishes. 


\section{$4.2 \mathrm{n}=2$}

Consider now the simplest nontrivial case of $c_{k} \propto \delta_{k n}$ for $n=2$, i.e. $A=A_{2}=\frac{1}{2} \mathcal{R} \circ L^{2}$. Applying twice (5), one gets

$$
\left(L^{2} \psi\right)_{i}=r_{i+2} r_{i+1} \psi_{i+2}+r_{i+1}\left(p_{i+1}+p_{i}\right) \psi_{i+1}+\left(R_{i+1}+p_{i}^{2}+R_{i}\right) \psi_{i}+r_{i}\left(p_{i}+p_{i-1}\right) \psi_{i-1}+r_{i} r_{i-1} \psi_{i-2}
$$

or

$$
\left(A_{2} \psi\right)_{i} \propto r_{i+2} r_{i+1} \psi_{i+2}+r_{i+1}\left(p_{i+1}+p_{i}\right) \psi_{i+1}-r_{i}\left(p_{i}+p_{i-1}\right) \psi_{i-1}-r_{i} r_{i-1} \psi_{i-2}
$$

while stationarity of the gradient of the trace part $\operatorname{Tr} L^{2}=\sum_{i}\left(p_{i}^{2}+2 R_{i}\right)$ in (31), which disappears from (32), lead exactly to the constraints (28) of the previous trivial example.

The commutativity constraints lead now to

$$
\begin{gathered}
R_{i+1}\left(p_{i+1}+p_{i}\right)=R_{i}\left(p_{i}+p_{i-1}\right) \\
R_{i+1}+p_{i}^{2}=R_{i-1}+p_{i-1}^{2}
\end{gathered}
$$

which reduce the dimension of the phase space to the four independent variables $\left(R_{i}\right.$ and $p_{i}$ on two neighboring sites).

Now, using (11), one can rewrite (32) in the basis of eigenfunctions of the Lax operator (5), e.g.

$$
A_{2}(\lambda)\left(\begin{array}{c}
\psi_{i} \\
\psi_{i-1}
\end{array}\right)=\left(\begin{array}{cc}
\frac{1}{2}\left(\lambda^{2}-p_{i}^{2}-R_{i+1}+R_{i}\right) & -r_{i}\left(\lambda+p_{i}\right) \\
r_{i}\left(\lambda+p_{i-1}\right) & -\frac{1}{2}\left(\lambda^{2}-p_{i-1}^{2}-R_{i-1}+R_{i}\right)
\end{array}\right)\left(\begin{array}{c}
\psi_{i} \\
\psi_{i-1}
\end{array}\right)
$$

In particular, upon (33) one gets $\operatorname{Tr} A_{2}(\lambda) \underset{(33)}{=} 0$.

For the operator (34) the equation of the curve (20) reads

$$
\begin{gathered}
y^{2}=\left(\lambda^{2}-\frac{1}{2} Q\right)^{2}-4 R_{i}\left(\lambda+p_{i}\right)\left(\lambda+p_{i-1}\right) \\
Q \equiv R_{i+1}+R_{i-1}-2 R_{i}+p_{i}^{2}+p_{i-1}^{2}
\end{gathered}
$$

The expansion of the generating differential at $\lambda \rightarrow \infty$ gives

$$
\begin{gathered}
y d \lambda \underset{\lambda \rightarrow \infty}{\sim} d \lambda\left(\lambda^{2}-\frac{1}{2}\left(Q+4 R_{i}\right)-\frac{2 R_{i}}{\lambda}\left(p_{i}+p_{i-1}\right)-\frac{R_{i}}{\lambda^{2}}\left(2 p_{i} p_{i-1}+Q+2 R_{i}\right)+\ldots\right)= \\
\underset{Q+4 R_{i}=2 C_{2}}{=} d \lambda\left(\lambda^{2}-C_{2}-\frac{2 C_{1}}{\lambda}-\frac{2 R_{i}}{\lambda^{2}}\left(p_{i} p_{i-1}-R_{i}+C_{2}\right)-\frac{2 C_{1} C_{2}}{\lambda^{3}}-\frac{2}{\lambda^{4}}\left(C_{1}^{2}+C_{2} H\right)+\ldots\right)
\end{gathered}
$$

where fixing $2 C_{2}=R_{i+1}+R_{i-1}+2 R_{i}+p_{i}^{2}+p_{i-1}^{2}$ together with the coefficient at $\frac{d \lambda}{\lambda}$ lead to the following Casimir constraints

$$
\begin{gathered}
C_{1}=R_{i}\left(p_{i}+p_{i-1}\right) \\
C_{2}=R_{i+1}+R_{i}+p_{i}^{2}=R_{i-1}+R_{i}+p_{i-1}^{2}
\end{gathered}
$$

which, in addition to (33) reduce the phase space to be two-dimensional. A convenient choice of co-ordinates is $R_{i}$ (for some fixed site $i$ ) and the difference between the corresponding momenta $p_{-}=p_{i}-p_{i-1}$.

The Hamiltonian $H$ is, up to a numeric factor, a coefficient at the $\frac{d \lambda}{\lambda^{2}}$-term in (36)

$$
H=R_{i}\left(p_{i} p_{i-1}-R_{i}+C_{2}\right) \underset{p_{i}-p_{i-1} \equiv p_{-}}{=} \frac{C_{1}^{2}}{4} \frac{1}{R_{i}}-\frac{1}{4} R_{i} p_{-}^{2}-R_{i}+C_{2} R_{i}
$$

With formulas (37) equation of the curve (35) acquires the form

$$
y^{2}=\left(\lambda^{2}-C_{2}\right)^{2}-4\left(C_{1} \lambda+H\right)
$$


and coincides exactly with the matrix model curve (3) for the potential $W=\frac{\lambda^{3}}{3}-C_{2} \lambda$ and function $f_{1}=C_{1} \lambda+H$.

Explicit calculation of the symplectic form for the $n=2$ case, using (23) and (19), gives

$$
\begin{gathered}
\Omega_{2}=\frac{1}{2} d\left(R_{i-1}+R_{i}+p_{i-1}^{2}\right) \wedge d \xi_{i-1}^{-}-\frac{1}{2} d\left(R_{i+1}+R_{i}+p_{i}^{2}\right) \wedge d \xi_{i}^{+}+d\left(p_{i}-p_{i-1}\right) \wedge d R_{i}= \\
\underset{(37)}{=} d\left(p_{i}-p_{i-1}\right) \wedge d R_{i}
\end{gathered}
$$

Now, in order to fit between the hamiltonian (38), the curve (39) and the symplectic form (40) one should rewrite equation (39) in the "Seiberg-Witten" form (see e.g. [13] and [11]), i.e. as

$$
\begin{gathered}
w+\frac{C_{1} \lambda+H}{w}=\lambda^{2}-C_{2} \\
w-\frac{C_{1} \lambda+H}{w}=y
\end{gathered}
$$

This means, in particular, that $2 w=y+\lambda^{2}-C_{2}$, and

$$
\frac{1}{2} d y \wedge d \lambda=d w \wedge d \lambda
$$

The first of the equations (41) can now be written as

$$
H=w \lambda^{2}-C_{1} \lambda-C_{2} w-w^{2}=w\left(\lambda-\frac{C_{1}}{2 w}\right)^{2}-\frac{C_{1}^{2}}{4 w}-C_{2} w-w^{2}
$$

which exactly coincides with (38), provided by the identifications

$$
\begin{gathered}
w \leftrightarrow-R_{i} \\
\lambda-\frac{C_{1}}{2 w} \leftrightarrow \pm p_{-}
\end{gathered}
$$

\section{$4.3 \mathrm{n}=3$}

For the $n=3$, with $c_{k} \propto \delta_{k 3}$ the explicit expression for the operator $A_{3}=\frac{1}{2} \mathcal{R} \circ L^{3}$ looks like

$$
\begin{gathered}
\left(A_{3} \psi\right)_{i} \propto r_{i+3} r_{i+2} r_{i+1} \psi_{i+3}+r_{i+2} r_{i+1}\left(p_{i+2}+p_{i+1}+p_{i}\right) \psi_{i+2}+ \\
+r_{i+1}\left(p_{i+1}^{2}+p_{i+1} p_{i}+p_{i}^{2}+r_{i+2}^{2}+R_{i+1}+R_{i}\right) \psi_{i+1}- \\
-r_{i}\left(p_{i}^{2}+p_{i} p_{i-1}+p_{i-1}^{2}+R_{i+1}+R_{i}+R_{i-1}\right) \psi_{i-1}- \\
\quad-r_{i-1} r_{i-2}\left(p_{i}+p_{i-1}+p_{i-2}\right) \psi_{i-2}-r_{i} r_{i-1} r_{i-2} \psi_{i-3}
\end{gathered}
$$

while stationarity of the gradient of the trace part

$$
\frac{1}{3} \operatorname{Tr} L^{3}=\frac{1}{3} \sum_{i} p_{i}^{3}+\sum_{i} p_{i}\left(R_{i}+R_{i+1}\right)
$$

gives rise to the constraints (33) of the previous example. The commutativity conditions (16) now give

$$
\begin{gathered}
p_{i}^{3}+\left(p_{i+1}+2 p_{i}\right) R_{i+1}+p_{i} R_{i}=p_{i-1}^{3}+\left(p_{i-2}+2 p_{i-1}\right) R_{i-1}+p_{i-1} R_{i} \\
R_{i+1}\left(p_{i+1}^{2}+p_{i+1} p_{i}+p_{i}^{2}+R_{i+2}+R_{i+1}+R_{i}\right)=R_{i}\left(p_{i}^{2}+p_{i} p_{i-1}+p_{i-1}^{2}+R_{i+1}+R_{i}+R_{i-1}\right)
\end{gathered}
$$

In the basis of eigenfunctions of the Lax operator matrix $A_{3}(\lambda)$ for (45) acquires the form

$$
\left(\begin{array}{cc}
\frac{1}{2}\left(\lambda^{3}+2 r_{i}^{2} \lambda-p_{i}^{3}-\left(p_{i+1}+2 p_{i}\right) r_{i+1}^{2}+p_{i-1} r_{i}^{2}\right) & -r_{i}\left(\lambda^{2}+p_{i} \lambda+p_{i}^{2}+r_{i+1}^{2}+r_{i}^{2}\right) \\
r_{i}\left(\lambda^{2}+p_{i-1} \lambda+p_{i-1}^{2}+r_{i}^{2}+r_{i-1}^{2}\right) & -\frac{1}{2}\left(\lambda^{3}+2 r_{i}^{2} \lambda-p_{i-1}^{3}-\left(p_{i-2}+2 p_{i-1}\right) r_{i-1}^{2}+p_{i} r_{i}^{2}\right)
\end{array}\right)
$$


For the operator (48) the curve (20) reads

$$
\begin{gathered}
y^{2}=\lambda^{6}-\left(p_{i}^{3}+p_{i-1}^{3}+\left(p_{i-2}+2 p_{i-1}\right) R_{i-1}+3\left(p_{i}+p_{i-1}\right) R_{i}+\left(p_{i+1}+2 p_{i}\right) R_{i+1}\right) \lambda^{3}- \\
-4 R_{i}\left(R_{i+1}+R_{i}+R_{i-1}+p_{i}^{2}+p_{i} p_{i-1}+p_{i-1}^{2}\right) \lambda^{2}-2 R_{i}\left(\left(p_{i+1}+2 p_{i}+2 p_{i-1}\right) R_{i+1}+\right. \\
\left.+\left(p_{i}+p_{i-1}\right) R_{i}+\left(p_{i-2}+2 p_{i-1}+2 p_{i}\right) R_{i-1}+p_{i}^{3}+2 p_{i}^{2} p_{i-1}+2 p_{i} p_{i-1}^{2}+p_{i-1}^{3}\right) \lambda \\
+\frac{1}{4}\left(\left(p_{i+1}+2 p_{i}\right) R_{i+1}-\left(p_{i}+p_{i-1}\right) R_{i}+\left(p_{i-2}+2 p_{i-1}\right) R_{i-1}+p_{i}^{3}+p_{i-1}^{3}\right)^{2}- \\
-4 R_{i}\left(p_{i}^{2}+R_{i+1}+R_{i}\right)\left(p_{i-1}^{2}+R_{i}+R_{i-1}\right)
\end{gathered}
$$

The expansion of the generating differential is

$$
\begin{gathered}
y d \lambda \underset{\lambda \rightarrow \infty}{\sim} d \lambda\left(\lambda^{3}-\frac{1}{2}\left(\left(p_{i+1}+2 p_{i}\right) R_{i+1}+3\left(p_{i}+p_{i-1}\right) R_{i}+\left(p_{i-2}+2 p_{i-1}\right) R_{i-1}+p_{i}^{3}+p_{i-1}^{3}\right)-\right. \\
-\frac{2 R_{i}}{\lambda}\left(R_{i+1}+R_{i}+R_{i-1}+p_{i}^{2}+p_{i} p_{i-1}+p_{i-1}^{2}\right)-\frac{R_{i}}{\lambda^{2}}\left(\left(p_{i+1}+2 p_{i}+2 p_{i-1}\right) R_{i+1}+\right. \\
\left.+\left(p_{i}+p_{i-1}\right) R_{i}+\left(p_{i-2}+2 p_{i-1}+2 p_{i}\right) R_{i-1}+p_{i}^{3}+2 p_{i}^{2} p_{i-1}+2 p_{i} p_{i-1}^{2}+p_{i-1}^{3}\right)- \\
-\frac{R_{i}}{\lambda^{3}}\left(\left(p_{i+1} p_{i}+p_{i+1} p_{i-1}+2 p_{i}^{2}+2 p_{i} p_{i-1}+2 p_{i-1}^{2}\right) R_{i+1}+\left(3 p_{i}^{2}+3 p_{i-1}^{2}+2 p_{i} p_{i-1}\right) R_{i}+\right. \\
+\left(p_{i-1} p_{i-2}+p_{i} p_{i-2}+2 p_{i} p_{i-1}+2 p_{i-1}^{2}+2 p_{i}^{2}\right) R_{i-1}+2\left(R_{i+1} R_{i}+R_{i+1} R_{i-1}+R_{i}^{2}+R_{i} R_{i-1}\right)+ \\
\left.\left.+p_{i}^{4}+p_{i}^{3} p_{i-1}+2 p_{i}^{2} p_{i-1}^{2}+p_{i} p_{i-1}^{3}+p_{i-1}^{4}\right)+\ldots\right)
\end{gathered}
$$

Fixing the coefficients at singular terms of the expansion of $y d \lambda$ to be the independent upon dynamical variables numbers (or "Casimirs"), using (48), one can write

$$
\begin{gathered}
C_{1}=R_{i}\left(p_{i}^{2}+p_{i} p_{i-1}+p_{i-1}^{2}+R_{i+1}+R_{i}+R_{i-1}\right) \\
C_{3}=p_{i}^{3}+\left(p_{i+1}+2 p_{i}\right) R_{i+1}+\left(p_{i-1}+2 p_{i}\right) R_{i}=p_{i-1}^{3}+\left(p_{i-2}+2 p_{i-1}\right) R_{i-1}+\left(2 p_{i-1}+p_{i}\right) R_{i}
\end{gathered}
$$

Together with (47), relations (51) impose four constraints to the eight-dimensional space of variables $\left(R_{j}\right.$ and $p_{j}$ with $j=i-1, i, i+1$ together with $R_{i-2}$ and $p_{i+2}$ ), so that the phase space in this example is four-dimensional.

Then the expansion (50) upon (51) turns into

$$
\begin{gathered}
y d \lambda \underset{\lambda \rightarrow \infty}{\sim} d \lambda\left(\lambda^{3}-C_{3}-\frac{2 R_{i}}{\lambda}\left(R_{i+1}+R_{i}+R_{i-1}+p_{i}^{2}+p_{i} p_{i-1}+p_{i-1}^{2}\right)-\right. \\
\begin{array}{c}
-\frac{2 R_{i}}{\lambda^{2}}\left(p_{i-1} R_{i+1}-\right. \\
\left.\left.=\left(p_{i}+p_{i-1}\right) R_{i}+p_{i} R_{i-1}+p_{i}^{2} p_{i-1}+p_{i} p_{i-1}^{2}+C_{3}\right)+\ldots\right)= \\
=d \lambda\left(\lambda^{3}-C_{3}-\frac{2 C_{1}}{\lambda}-\frac{2 H_{1}}{\lambda^{2}}-\frac{2 H_{2}}{\lambda^{3}}+\ldots\right)
\end{array}
\end{gathered}
$$

so that the first of two independent hamiltonians (proportional to the coefficients at $\frac{d \lambda}{\lambda^{2}}$ and $\frac{d \lambda}{\lambda^{3}}$ ) can be, using (51), presented as

$$
H_{1}=C_{3} R_{i}+\frac{C_{1}}{2}\left(p_{i}+p_{i-1}\right)-\frac{1}{2} R_{i}\left(p_{i}^{3}+p_{i-1}^{3}+3 R_{i}\left(p_{i}+p_{i-1}\right)+\left(p_{i}-p_{i-1}\right)\left(R_{i+1}-R_{i-1}\right)\right)
$$

and

$$
\begin{aligned}
& H_{2}=\frac{C_{1}^{2}}{4 R_{i}}+\frac{C_{1}}{2}\left(R_{i}-p_{i} p_{i-1}\right)+C_{3} R_{i}\left(p_{i}+p_{i-1}\right)+\frac{1}{4} R_{i}^{3}- \\
& -\frac{1}{4} R_{i}\left(R_{i+1}-R_{i-1}\right)^{2}-\frac{1}{2} R_{i}\left(R_{i+1}-R_{i-1}\right)\left(p_{i}^{2}-p_{i-1}^{2}\right)- \\
& -\frac{1}{4} R_{i}\left(p_{i}^{4}+3 p_{i}^{2} p_{i-1}^{2}+p_{i-1}^{4}\right)-R_{i}^{2}\left(p_{i}^{2}+\frac{5}{3} p_{i} p_{i-1}+p_{i-1}^{2}\right)
\end{aligned}
$$

The equation of the curve (49) now becomes

$$
y^{2}=\left(\lambda^{3}-C_{3}\right)^{2}-4\left(C_{1} \lambda^{2}+H_{1} \lambda+H_{2}\right)
$$


again coinciding with (3) with the potential $W=\frac{\lambda^{4}}{4}-C_{3} \lambda$ and function $f_{2}=C_{1} \lambda^{2}+H_{1} \lambda+H_{2}$.

Explicit calculation of the symplectic form $\Omega_{3}$ gives

$$
\begin{gathered}
\Omega_{3}=\frac{1}{2} d \xi_{i-1}^{-} \wedge d\left(p_{i-1}^{3}+\left(p_{i-2}+2 p_{i-1}\right) R_{i-1}+\left(p_{i}+2 p_{i-1}\right) R_{i}\right)+ \\
+\frac{1}{2} d\left(p_{i}^{3}+\left(p_{i+1}+2 p_{i}\right) R_{i+1}+\left(p_{i-1}+2 p_{i}\right) R_{i}\right) \wedge d \xi_{i}^{+} \\
+d\left(R_{i+1}-R_{i-1}\right) \wedge d R_{i}+2 R_{i} d p_{i} \wedge d p_{i-1}+ \\
+d\left(p_{i}^{2}-p_{i-1}^{2}\right) \wedge d R_{i}+\left(p_{i-1} d p_{i}-p_{i} d p_{i-1}\right) \wedge d R_{i}
\end{gathered}
$$

Using (51), and introducing $R_{-} \equiv R_{i+1}-R_{i-1}$ this slightly simplifies to

$$
\begin{gathered}
\Omega_{3}=d R_{-} \wedge d R_{i}+2 R_{i} d p_{i} \wedge d p_{i-1}+ \\
+d\left(p_{i}^{2}-p_{i-1}^{2}\right) \wedge d R_{i}+\left(p_{i-1} d p_{i}-p_{i} d p_{i-1}\right) \wedge d R_{i}
\end{gathered}
$$

(certainly, $\left.d\left(\Omega_{3}\right)=0\right)$, or

$$
\Omega_{3}=\left(d R_{-} d R_{i} d p_{i} d p_{i-1}\right) \cdot \hat{\Omega}_{3} \cdot\left(\begin{array}{c}
d R_{-} \\
d R_{i} \\
d p_{i} \\
d p_{i-1}
\end{array}\right)
$$

where the wedge product is implied and $\hat{\Omega}_{3}$ is the matrix

$$
\hat{\Omega}_{3}=\left(\begin{array}{cccc}
0 & \frac{1}{2} & 0 & 0 \\
-\frac{1}{2} & 0 & -\left(p_{i}+\frac{p_{i-1}}{2}\right) & p_{i-1}+\frac{p_{i}}{2} \\
0 & p_{i}+\frac{p_{i-1}}{2} & 0 & R_{i} \\
0 & -\left(p_{i-1}+\frac{p_{i}}{2}\right) & -R_{i} & 0
\end{array}\right)
$$

A nontrivial check is that the Hamiltonians (53) and (54) indeed commute

$$
\left\{H_{1}, H_{2}\right\}_{\Omega_{3}}=0
$$

with respect to the Poisson bracket, corresponding to symplectic structure (57) or defined by the inverse to (59) matrix

$$
\left(\begin{array}{cccc}
0 & -2 & \frac{2 p_{i-1}+p_{i}}{R_{i}} & \frac{2 p_{i}+p_{i-1}}{R_{i}} \\
2 & 0 & 0 & 0 \\
-\frac{2 p_{i-1}+p_{i}}{R_{i}} & 0 & 0 & -\frac{1}{R_{i}} \\
-\frac{2 p_{i}+p_{i-1}}{R_{i}} & 0 & \frac{1}{R_{i}} & 0
\end{array}\right)
$$

giving rise to the "elementary brackets"

$$
\begin{gathered}
\left\{R_{i}, R_{-}\right\}_{\Omega_{3}}=1 \\
\left\{p_{i}, p_{i-1}\right\}_{\Omega_{3}}=-\frac{1}{2 R_{i}} \\
\left\{R_{-}, p_{i}\right\}_{\Omega_{3}}=\frac{1}{R_{i}}\left(p_{i-1}+\frac{p_{i}}{2}\right) \\
\left\{R_{-}, p_{i-1}\right\}_{\Omega_{3}}=\frac{1}{R_{i}}\left(p_{i}+\frac{p_{i-1}}{2}\right)
\end{gathered}
$$


The symplectic structure (57) can be brought to canonical form, introducing new variables as $p_{i}=p \cosh \theta$ and $p_{i-1}=p \sinh \theta$, then

$$
\begin{gathered}
\Omega_{3}=d R_{-} \wedge d R_{i}+R_{i} d\left(p^{2}\right) \wedge d \theta+p^{2} d R_{i} \wedge d \theta+d\left(p^{2}\right) \wedge d R_{i}= \\
=d\left(R_{-}+p^{2}\right) \wedge d R_{i}+d\left(R_{i} p^{2}\right) \wedge d \theta
\end{gathered}
$$

and one concludes that the Darboux co-ordinates are $\theta, R \equiv R_{i}$ together with

$$
\begin{gathered}
\rho=R_{i} p^{2} \\
\Delta=R_{-}+p^{2}
\end{gathered}
$$

In these variables the Poisson brackets are

$$
\{\Delta, R\}_{\Omega_{3}}=1, \quad\{\rho, \theta\}_{\Omega_{3}}=1
$$

while all other vanish. The Hamiltonians (53), (54) in these variables have the form

$$
\begin{gathered}
H_{1}=C_{3} R+\frac{C_{1}}{2} \rho^{1 / 2} R^{-1 / 2} e^{\theta}-\frac{1}{2} \Delta \rho^{1 / 2} R^{1 / 2} e^{-\theta}+\frac{1}{8}\left(e^{-\theta}-e^{3 \theta}\right) \rho^{3 / 2} R^{-1 / 2}-\frac{3}{2} \rho^{1 / 2} R^{3 / 2} e^{\theta} \\
H_{2}=\frac{C_{1}^{2}}{4 R}+\frac{C_{1}}{2} R+\frac{C_{1}}{8} \frac{\rho}{R}\left(e^{-2 \theta}-e^{2 \theta}\right)+C_{3} \rho^{1 / 2} R^{1 / 2} e^{\theta}+\frac{1}{4} R^{3}-\frac{1}{4} \Delta^{2} R-\frac{1}{32} \frac{\rho^{2}}{R}-\frac{9}{8} \rho R e^{2 \theta}+ \\
+\frac{1}{8} \rho R e^{-2 \theta}+\frac{1}{64} \frac{\rho^{2}}{R}\left(e^{4 \theta}+e^{-4 \theta}\right)
\end{gathered}
$$

i.e. are generally functions of the fractional powers of dynamical variables. The dependence on fractional powers, however, disappears for $H_{2}$ at vanishing Casimir $C_{3}=0$ and for the square $H_{1}^{2}$ at $C_{1}=C_{3}=0$.

\section{Discussion}

Let us, finally, discuss some related issues and open problems.

\section{Geometry on stationary and periodic problems}

When defining the stationary problem, we have started with an infinite Toda chain. Remaining all ingredients almost intact, one could take as initial point a $N$-periodic problem in Toda instead $\left(q_{i+N}=q_{i}, p_{i+N}=p_{i}\right.$, $\psi_{i+N}=w \psi_{i}$ etc) with sufficiently large $N$.

Such procedure, however, may be already treated purely in terms of algebro-geometric integrable systems. One may start with the curve of $N$-periodic chain

$$
w+\frac{1}{w}=P_{N}(\lambda)
$$

endowed with generating differential $d S=\lambda \frac{d w}{w}$ and consider its reduction down to $2 n$-dimensional subspace in the moduli space for $n<N$. A natural way to impose such reduction is to require existence on the curve (67) of a single-valued meromorphic function with only two poles $($ at $\lambda=\infty$ ) of order $n$. Existence of such function on the curve (67) is exactly equivalent to stationarity of the order $n$ flow in periodic Toda, associated with the linear combination of meromorphic differentials $d \Omega_{A}=\sum_{k=1}^{n} c_{k} d \Omega_{k}$. Since there is no natural function with desired properties on the curve (67) in general position, this constraint effectively reduces the (smooth) genus of the Riemann surface from $N-1$ to $n-1$ and one ends up with the curve of a stationary Toda chain (3). Such reduction was already discussed in the literature comparing the Seiberg-Witten and Dijkgraaf-Vafa geometries, see [3] and, for example, $[14,15,16]$. Note also, that the corresponding generating differentials or symplectic structures remain unrelated by this procedure, and this exactly corresponds to the difference between the symplectic structures (21), (40) and (57) of stationary problem and that of the infinite chain we discussed above. 


\section{Remarks on quantum case}

For the curve (39) and the symplectic form (42) one can immediately write the "naive" Schrödinger equation

$$
\hbar^{2} \frac{\partial^{2}}{\partial \lambda^{2}} \Upsilon=\left(W^{\prime}(\lambda)^{2}-\hbar W^{\prime \prime}(\lambda)\right) \Upsilon
$$

with the solution

$$
\Upsilon=\exp \left(-\frac{1}{\hbar} W(\lambda)\right)
$$

since

$$
\hbar^{2} \frac{\partial^{2}}{\partial \lambda^{2}}-W^{\prime}(\lambda)^{2}+\hbar W^{\prime \prime}(\lambda)=\left(\hbar \frac{\partial}{\partial \lambda}-W^{\prime}(\lambda)\right)\left(\hbar \frac{\partial}{\partial \lambda}+W^{\prime}(\lambda)\right)
$$

Comparison of (68) with (39) for the potential $W^{\prime}(\lambda)=\lambda^{2}-C_{2}$ gives $H=0$ and $C_{1}=\frac{\hbar}{2}$, and comparison with (55) leads to $H_{2}=H_{1}=0$ and $C_{1}=\frac{3}{4} \hbar$. Formulas (41) and (42) rather suggest that the quantization can be better performed in the $(y, w)$-variables. For the periodic Toda chain (67) this way leads to the Baxter second-order difference equation, but for the stationary problem equation (41), due to (42) would give rise to an integral operator. The main obstacle on this way is that it is vague what are the "proper" variables for quantization of stationary problem, since using the naive Darbough co-ordinates, already in $n=3$ case, leads to the non-analytic dependencies in the Hamiltonians.

One can nevertheless try to impose the quantum relations

$$
\hat{\mathcal{H}}_{i} \mathcal{Z}=H_{i} \mathcal{Z}, \quad i=1, \ldots, n-1
$$

onto the matrix model $\mathcal{Z}$-function, which instead of naive partition function $Z$ defined by integral representation (1) contains explicitly information about (quantum version of) geometry (3), (4), i.e. one literally has to solve (71) for the function

$$
\mathcal{Z}(c, H)=\exp \left(\frac{1}{\hbar^{2}} F_{0}(c, H)+\ldots\right)
$$

This formulation of quantum problem is consistent with the fact that the form of Hamiltonians (66) simplifies at vanishing (clasically) Casimirs, when they are of order of $\hbar$ or being some quantum corrections. The classical formulas (66) for $H_{i}$ (or some polynomial functions of them) are then replaced by differential and difference operators $\hat{\mathcal{H}}_{i}$ via $\Delta \rightarrow \partial / \partial R$ and $\theta \rightarrow \partial / \partial \rho$, so that $e^{ \pm \theta}$ turn into the shift operators in $\rho$-variable. Constructed in this way $\mathcal{Z}$-function $(72)$ can be treated as a matrix-model analogue of Nekrasov's generalization of the Seiberg-Witten prepotential [17].

\section{Acknowledgements}

I am grateful to H. Braden, R. Donagi, H. Kanno, S. Kharchev, B. Khesin, A. Mironov, N. Nekrasov, M. Olshanetsky, V. Rubtsov, A. Veselov and, especially, to I. Krichever for very useful discussions. The work was partially supported by the RFBR grant 04-01-00642, the grant for support of scientific schools 1578.2003.2, the Federal Program of the Russian Ministry of Industry, Science and Technology No 40.052.1.1.1112, and the Russian Science Support Foundation.

\section{References}

[1] A. A. Migdal, Phys. Rept. 102, 199 (1983).

[2] F. David, Phys.Lett. B302 (1993) 403-410, arXiv:hep-th/9212106;

G. Bonnet, F. David, B. Eynard, J.Phys. A33 (2000) 6739-6768, arXiv:cond-mat/0003324. 
[3] F. Cachazo, K. Intriligator and C. Vafa, Nucl.Phys. B603 (2001) 3-41; arXiv:hep-th/0103067;

F. Cachazo and C. Vafa, arXiv:hep-th/0206017.

[4] R. Dijkgraaf and C. Vafa, arXiv:hep-th/0206255; arXiv:hep-th/0207106; arXiv:hep-th/0208048.

[5] I. Krichever, Commun. Pure. Appl. Math. 47 (1992) 437, hep-th/9205110.

[6] A. Gerasimov, A. Marshakov, A. Mironov, A. Morozov and A. Orlov, Nucl. Phys. B 357 (1991) 565;

S. Kharchev, A. Marshakov, A. Mironov, A. Orlov and A. Zabrodin, Nucl. Phys. B 366 (1991) 569.

[7] A. Gorsky, I. Krichever, A. Marshakov, A. Mironov and A. Morozov, Phys.Lett., B355 (1995) 466-477, hep-th/9505035.

[8] O. I. Bogoyavlensky and S. P. Novikov, Func. Anal. Appl. 10 (1976) 9;

S. Novikov, S. Manakov, L. Pitaevsky and V. Zakharov, Theory of solitons, Moscow, Nauka 1980;

B.Dubrovin, I.Krichever and S.Novikov, Integrable systems - I, Sovremennye problemy matematiki (VINITI), Dynamical systems - 4 (1985) 179.

[9] I. M. Krichever and D. H. Phong, J. Diff. Geom. 45 (1997) 349 [arXiv:hep-th/9604199]; arXiv:hepth/9708170.

[10] A. Marshakov, in Proceedings of 10th International Conference "Problems of Quantum Field Theory", Dubna 1996, arXiv:hep-th/9607159.

[11] A. Marshakov, Seiberg-Witten Theory and Integrable Systems, World Scientific, 1999.

[12] Integrability: The Seiberg-Witten and Whitham Equations Ed. by H. W. Braden and I. M. Krichever. Gordon and Breach, 2000.

[13] A. Marshakov, Mod. Phys. Lett. A 11 (1996) 1169 [arXiv:hep-th/9602005];

A. Gorsky, A. Marshakov, A. Mironov and A. Morozov, Phys. Lett. B 380 (1996) 75 [arXiv:hep-th/9603140].

[14] T. J. Hollowood, JHEP 0310 (2003) 051 [arXiv:hep-th/0305023].

[15] R. Boels, J. de Boer, R. Duivenvoorden and J. Wijnhout, JHEP 0403 (2004) 010 [arXiv:hep-th/0305189].

[16] H. Itoyama and H. Kanno, Nucl. Phys. B 686 (2004) 155 [arXiv:hep-th/0312306].

[17] N. A. Nekrasov, Adv. Theor. Math. Phys. 7 (2004) 831 [arXiv:hep-th/0206161], arXiv:hep-th/0412021;

A. S. Losev, A. Marshakov and N. A. Nekrasov, arXiv:hep-th/0302191;

N. Nekrasov and A. Okounkov, arXiv:hep-th/0306238;

see also

A. Braverman, math.AG/0401409;

A. Braverman and P. Etingof, math.AG/0409441. 